
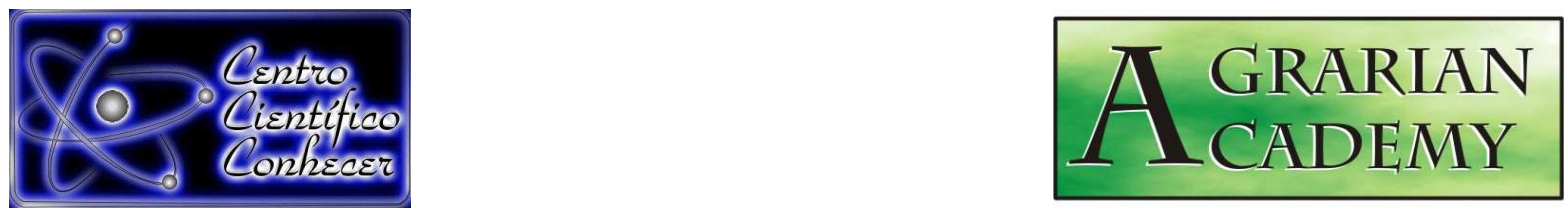

\title{
CHECKLIST DAS ESPÉCIES DE ANGIOSPERMAS DOADAS SOB ACERVO, NA COLEÇÃO BOTÂNICA DO LABORATÓRIO DE BIOLOGIA VEGETAL, DA UNIVERSIDADE ESTADUAL DO MARANHÃO
}

\author{
Silmara Gomes Queirós ${ }^{1}$, Gonçalo Mendes da Conceição ${ }^{2}$, Janilde de Melo \\ Nascimento ${ }^{3}$ \\ ${ }^{1}$ Graduanda em Ciências Biológicas Licenciatura, da \\ Universidade Estadual do Maranhão (silmara.queiros@bol.com.br) \\ ${ }^{2}$ Professor Doutor do Centro de Estudos Superiores de Caxias/CESC/UEMA \\ ${ }^{3}$ Mestre em Botânica Tropical UFRA/MPEG \\ Caxias-Brasil
}

Recebido em: 03/01/2014 - Aprovado em: 04/04/2014 - Publicado em: 12/04/2014

\section{RESUMO}

Os herbários são coleções de plantas secas que documentam a diversidade vegetal de determinada região ou país. Além de indispensáveis para estudos de sistemática de plantas os herbários também funcionam como ferramentas de apoio à pesquisa para muitas outras áreas do conhecimento. Atualmente o Index Herbariorum lista para o mundo aproximadamente 3.400 herbários, sendo 121 o número herbários ativos no Brasil e três no estado do Maranhão. O presente trabalho teve por objetivos realizar um levantamento de espécimes oriundos de intercâmbio acervados no Herbário Professor Aluízio Bittencourt, do Centro de Estudos Superiores de Caxias, da Universidade Estadual do Maranhão. Os dados foram obtidos mediante revisão do acervo levantando-se o local de origem, família, gênero e espécie de cada espécime depositado no Herbário. Dos 4.135 espécimes acervados, 645 são provenientes de doações, estando distribuídos em 90 famílias, 223 gêneros e 391 espécies. O presente resultado reflete a importância da atividade de intercâmbio para a ampliação e aprimoramento das coleções botânicas.

PALAVRAS- CHAVE: coleções, Herbário, Intercâmbio

\section{CHECKLIST OF SPECIES ANGIOSPERM DONATED ASSETS UNDER, THE COLLECTION OF BOTANICAL LABORATORY PLANT BIOLOGY, THE STATE UNIVERSITY OF MARANHÃO}

\begin{abstract}
The Herbaria are collections of dried plants documenting plant diversity in a region or country. Besides indispensable for systematic studies of the herbal plants also function as research support for many other areas of knowledge tools. Currently the Index Herbarium list for the world approximately 3.400 herbaria, 121 the active herbaria in Brazil and number 3 in the state of Maranhão. This research aimed to conduct a survey of specimens derived from the Herbarium exchange acervados Teacher Aluízio Bittencourt Center for Advanced Studies in Caxias, State University of Maranhão. The data were obtained by review of the acquis rising place of origin, family, genus and species of each specimen deposited in the herbarium. Of 4. Acervados 135 specimens, 645 are from donations, being distributed in 90 families and 391 species 223 gêner. The results reflect the importance of interchange activity for the expansion and improvement of botanical collections.
\end{abstract}


KEYWORDS: Herbarium, Exchange, Collections.

\section{INTRODUÇÃO}

Herbário é uma coleção de plantas secas, preparadas técnica e cientificamente para posteriores estudos, seja ele comparativo, histórico ou documentário da flora de determinada região ou país (RESENDE \& GUIMARÃES, 2007). Os espécimes depositados nesses espaços servem como registros da variação morfológica e genética, distribuição geográfica, bem como de outras valiosas informações, sendo muitas vezes o único registro de uma espécie extinta ou de espécies vistas na natureza apenas uma vez em sua forma selvagem (MANIA \& ASSIS, 2008).

Atualmente o Index Herbariorum lista para o mundo aproximadamente 3.400 herbários, compreendendo cerca de 350 milhões de exemplares que documentam a vegetação da terra nos últimos 400 anos (THIERS, 2014).

O Brasil conta hoje com 226 Herbários, dos quais 121 encontram-se ativos, 100 inativos e cinco estão em processo de transferência (SOCIEDADE BOTÂNICA DO BRASIL, 2013). Destes pelo menos 87 estão registrados no Index Herbariorum, onde o número estimado de exemplares depositados nas coleções botânicas brasileiras é de aproximadamente 5,1 milhões (BARBOSA \& VIEIRA, 2005). Grande parte dos Herbários brasileiros encontram-se nas regiões Sul, Sudeste e Nordeste do país (PEIXOTO \& MORIM, 2003), por outro lado as regiões Norte e Centro-Oeste são as que detêm os menores números de herbários e de espécimes depositados (BARBOSA \& VIEIRA, 2005).

Para o estado do Maranhão estão registrados três Herbários ativos, um destes, Herbário Aluísio Bittencourt (HABIT), localizado no Centro de Estudos Superiores de Caxias (CESC), da Universidade Estadual do Maranhão (UEMA), na cidade de Caxias, área de estudo em questão (SOCIEDADE BOTÂNICA DO BRASIL, 2013) com número estimado em 4. 315 espécimes depositados.

Nos Herbários encontram-se depositados materiais e dados que certificam sobre a diversidade e a riqueza da flora de determinada região ou país (PEIXOTO et al., 2009). Além de indispensáveis para estudos de sistemática de plantas, os Herbários também funcionam como ferramentas de apoio à pesquisa para muitas outras áreas do conhecimento (PEIXOTO \& BARBOSA, 1989 citado por PEIXOTO et al., 2009). O conjunto de espécimes de cada Herbário reflete também parte do histórico da instituição e também dos botânicos e administradores que por ela passaram (PEIXOTO et al., 2009).

Além do desenvolvimento de pesquisas, o Herbário também mantém vínculos de colaboração com unidades similares em todo o mundo, como o intercâmbio de materiais e apoio de especialistas (PEIXOTO et al., 2009). O intercâmbio consiste no empréstimo, troca ou doação de materiais científicos e dados, com o intuito de identificação de espécimes ou ampliação do acervo (MACHADO \& BARBOSA, 2010). Entre Herbários do Brasil e destes com Herbários do exterior é uma das atividades de rotina que muito enriquece as coleções e auxilia nos processos de identificações, aumentando a qualidade dos espécimes depositados (PEIXOTO et al., 2009).

Os Herbários podem conter em suas coleções representantes da flora local, regional, nacional ou mesmo mundial, e de significância histórica. Nos Herbários são obtidos ainda, os dados básicos sobre cada espécie, como a distribuição geográfica, aspectos fenológicos e outras informações. Neste sentido, 
no presente trabalho teve por objetivo elaborar checklist das espécies de angiospermas doadas para o acervo da Coleção Botânica, do Laboratório de Biologia Vegetal, do Centro de Estudos Superiores de Caxias/CESC, da Universidade Estadual do Maranhão/UEMA.

\section{MATERIAL E MÉTODOS}

O Herbário Prof. Aluísio Bittencourt (HABIT), encontra-se localizado nas dependências do Laboratório de Biologia Vegetal (LABIVE), do Centro de Estudos Superiores de Caxias, da Universidade Estadual do Maranhão (CESC/UEMA), possuindo atualmente um acervo com 4. 135 exsicatas.Fundado em agosto de 1991 pelo Prof. Deusiano Bandeira de Almeida, inicialmente com funcionamento nas dependências do Laboratório de Anatomia e nomeado de Herbário Aylton Brandão Joly, em homenagem a um dos grandes nomes da Botânica no Brasil.

Para obtenção dos dados foram realizadas três etapas: na primeira etapa foi feita a revisão das exsicatas do acervo separando-se e catalogando-se os espécimes doados. Na segunda etapa foram levantados os locais de procedência de cada um dos espécimes e quantificado o número de espécimes para cada local de origem. Em uma terceira etapa foi realizado o levantamento em nível de família, gênero e espécie dos espécimes, identificando-se a família botânica com maior número de representantes dentre os materiais analisados, confirmando-se a nomenclatura com auxílio de material bibliográfico especializado e fazendo as devidas correções quando necessário com base na LISTA DE ESPÉCIES DA FLORA DO BRASIL (2014) e TROPICUS (2014).

\section{RESULTADOS E DISCUSSÃO}

Durante a revisão do acervo foram catalogados 4.135 espécimes acervados, dentre os quais 645 foram doados ao Herbário, sendo registrados 20 locais de procedência distintos, com a predominância de espécimes provenientes do Distrito Federal, seguido de Balsas e Goiás (Quadro 1), (Figura 2 e 3). Dentre os espécimes doados quatro espécimes vieram de instituições estrangeiras, sendo uma da Argentina: Brassaia actinophylla (Araliaceae) e três do Paraguai, sendo uma espécime de Allophylus edulis (Sapindaceae) e duas espécimes de Vernonia bardanoides (Asteraceae), (Figura 1). Os espécimes encontram-se distribuídos em 90 famílias, 223 gêneros e 391 espécies. A família Fabaceae destacou-se entre todas as demais famílias por apresentar um maior representativo tanto de gêneros e espécies como de espécimes, aproximando-se desta apenas a família Poaceae, seguida da família Asteraceae e Myrtaceae (Quadro 2 e Tabela 1), (Figura 4).

$\mathrm{O}$ intercâmbio entre Herbários vem difundindo o conhecimento sobre a flora, isso por intermédio de doações ou troca de materiais entre estas instituições. Grande parte dos Herbários tem ampliado suas coleções, graças à atividade continua de intercambio entre estas, e isso vem sendo refletido não somente no número de espécimes acervados, mas na abrangência das coleções, que passam a representar não somente a diversidade da flora local, mas regional, nacional e mundial como é o caso dos maiores herbários do mundo (PEIXOTO \& MORIM, 2003).

Conforme ressalta BARBOSA \& VIEIRA (2005), dentre os Herbários brasileiros 125 estão ativos em intercâmbio de dados e materiais científicos. A atividade de intercâmbio entre Herbários brasileiros e destes com instituições estrangeiras além de ampliar o acervo, vêm também auxiliar no processo de 
identificação dos espécimes (PEIXOTO \& MORIM, 2003), isso por intermédio da inserção de novas espécies as coleções.

O Herbário IBGE é um dentre os Herbários brasileiros que mantêm constante desde a sua fundação, intercâmbio tanto com instituições nacionais, compreendendo 80 Herbários como internacionais, 100 Herbários e cerca de 1.400 taxonomistas envolvidos (RESENDE \& GUIMARÃES, 2007). Outro Herbário brasileiro que também vem desempenhando esse tipo de atividade é o Herbário ICN, da Universidade Federal do Rio Grande do Sul, que tem mantido um fluxo constante de materiais que tanto são enviados para identificação, como na forma de permutas, empréstimos ou doações (HERBÁRIO ICN, 2014).

De acordo com PEIXOTO \& MORIM (2003), o aumento da representatividade das espécies brasileiras em coleções botânicas do país, assim bem como a preservação destas, são elementos essências para o conhecimento da flora nacional.

QUADRO 1. Distribuição do número de espécimes doados ao Herbário Prof. Aluísio Bittencourt, de acordo com o local de procedência.

\begin{tabular}{|l|l|}
\hline \multicolumn{1}{|c|}{ LOCAL DE ORIGEM } & \multicolumn{1}{c|}{$\mathbf{N}^{\circ}$ DE ESPÉCIMES } \\
\hline BRASÍLIA (DF) & 318 \\
\hline BALSAS (MA) & 73 \\
\hline GOIÁS (AL) & 31 \\
\hline MINAS GERAIS (MG) & 21 \\
\hline BAHIA (BA) & 17 \\
\hline SÃO PAULO (SP) & 13 \\
\hline MATO GROSSO (MT) & 10 \\
\hline MATO GROSSO DO SUL (MS) & 4 \\
\hline PARÁ (PA) & 3 \\
\hline TOCANTINS (TO) & 3 \\
\hline PARAGUAI & 3 \\
\hline CAROLINA (MA) & 3 \\
\hline RONDÔNIA (RO) & 2 \\
\hline SERGIPE (AL) & 1 \\
\hline AMAZONAS (AM) & 1 \\
\hline MACEIÓ (AL) & 1 \\
\hline CEARÁ (CE) & 1 \\
\hline ARGENTINA & 13 \\
\hline RIO GRANDE DO NORTE (RN) & 13 \\
\hline SANTA QUITÉRIA (MA) & 13 \\
\hline
\end{tabular}


QUADRO 2. Lista de famílias e espécies doadas ao Herbário Prof. Aluísio Bittencourt, do Centro de Estudos Superiores de Caxias/CESC-UEMA.

\begin{tabular}{|c|c|}
\hline FAMÍLIA & ESPÉCIES \\
\hline ACANTHACEAE & $\begin{array}{l}\text { - Aphelandra squarrosa Nees } \\
\text { - Justicia L. } \\
\text { - Ruellia L. } \\
\text { - Stenandrium Nees. } \\
\text { - Stenandrium pohlii Nees. }\end{array}$ \\
\hline ALSTROMERIACEAE & - Bomarea hirta Schenk \\
\hline AMARANTHACEAE & $\begin{array}{l}\text { - Gomphrena rudis L. } \\
\text { - Gomphrena arborescens L.f. }\end{array}$ \\
\hline AMARYLLIDACEAE & - Crinum (L.) \\
\hline ANEMIACEAE & $\begin{array}{l}\text {-Anemia (L.) Sw. } \\
\text {-Anemia phyllitidis (L.) Sw. }\end{array}$ \\
\hline ANNONACEAE & $\begin{array}{l}\text { - Cardiopetalum calophyllum Schltdl. } \\
\text {-Xylopia L. } \\
\text { - Xylopia sericea A.St.-Hil. } \\
\text { - Guatteria sellowiana Schltdl. } \\
\text { - Xylopia aromatica (Lam.) Mart. } \\
\text { - Annona L. }\end{array}$ \\
\hline APOCYNACEAE & - Aspidosperma tomentosum Mart. \\
\hline ARACEAE & $\begin{array}{l}\text { - Syagrus comosa (Mart.). Mart. } \\
\text { - Astrocaryum G. Mey }\end{array}$ \\
\hline ARALIACEAE & $\begin{array}{l}\text { - Brassaia actinophylla Endl. } \\
\text { - Didymopanax Decne. \& Planch. } \\
\text { - Didymopanax macrocarpum (Cham. \& Schltdl.) } \\
\text { Seem. }\end{array}$ \\
\hline ARECACEAE & - Astrocaryum G.Mey. \\
\hline \multirow[t]{2}{*}{ ARISTOLOCHIACEAE } & $\begin{array}{l}\text { - Aristolochia L. } \\
\text { - Aristolochia galeata Mart. \& Zucc. }\end{array}$ \\
\hline & $\begin{array}{l}\text { - Centratherum Cass. } \\
\text { - Cotula L. }\end{array}$ \\
\hline
\end{tabular}


QUADRO 2. Cont. Lista de famílias e espécies doadas ao Herbário Prof. Aluísio Bittencourt, do Centro de Estudos Superiores de Caxias/CESC-UEMA.

\begin{tabular}{|c|c|}
\hline ASTERACEAE & $\begin{array}{l}\text { - Ichthyothere cunabi Mart. } \\
\text { - Mikania psilostachya DC. } \\
\text { - Mikania smilacina DC. } \\
\text { - Austrobrickellia R. M. King \& H. Rob. } \\
\text { - Piptocarpha rotundifolia (Less.). Baker } \\
\text { - Praxelis kleinioides (Kunth) Sch. Bip. } \\
\text { - Stilpnopappus scaposus DC. } \\
\text { - Vernonia Schreb. } \\
\text { - Vernonia apiculata Mart. ex, DC. } \\
\text { - Vernonia aurea Mart. ex, DC. } \\
\text { - Vernonia bardanoides Less. } \\
\text { - Vernonia dura Mart. ex, DC. } \\
\text { - Vernonia ferruginea Less. } \\
\text { - Vernonia erythrophila DC. } \\
\text { - Vernonia glandulosa DC. } \\
\text { - Vernonia obtusa (Gleason) S.F.Blake } \\
\text { - Vernonia polyanthes (Spreng.). Less. } \\
\text { - Vernonia remotiflora Rich. } \\
\text { - Vernonia rubriaamea Mart. ex, DC. } \\
\text { - Vernonia zuccariniana Mart. ex, DC. } \\
\text { - Wulffia sp. Neck. } \\
\text { - Wulffia stenoglossa (DC.). Huber }\end{array}$ \\
\hline BIGNONIACEAE & $\begin{array}{l}\text { - Arrabidaea brachypoda (DC.). Bureau } \\
\text { - Arrabidaea sceptrum (Cham.). Bandwidth } \\
\text { - Jacaranda ulei Bureau \& K.Schum. } \\
\text { - Zeyheria sp. Mart. } \\
\text { - Zeyheria digitalis (Vell.) L.B. Sm. \& Sandwith }\end{array}$ \\
\hline BIXACEAE & $\begin{array}{l}\text { - Bixa orellana L. } \\
\text { - Cochlospermum orinocense (Kunth) Steud. } \\
\text { - Cochlospermum regium (Schrank) Pilg. }\end{array}$ \\
\hline
\end{tabular}


QUADRO 2. Cont. Lista de famílias e espécies doadas ao Herbário Prof. Aluísio Bittencourt, do Centro de Estudos Superiores de Caxias/CESC-UEMA.

\begin{tabular}{|c|c|}
\hline BLECHNACEAE & - Blechnum occidentale L. \\
\hline BORAGINACEAE & $\begin{array}{l}\text { - Cordia caleocephala Cham. } \\
\text { - Cordia multispicata Cham. } \\
\text { - Cordia trichotoma (Vell.) } \\
\text { - Tournefortia paniculata Cham. }\end{array}$ \\
\hline BURSERACEAE & $\begin{array}{l}\text { - Almecega Marchand. } \\
\text { - Bursera Jacq. ex L. } \\
\text { - Protium almecega Marchand. } \\
\text { - Protium ovatum Engl. }\end{array}$ \\
\hline CALOPHYLLACEAE & - Kielmeyera Mart. \& Zucc. \\
\hline CAMPANULACEAE & - Siphocampylus lycioides (Cham.) G. Don \\
\hline CELASTRACEAE & $\begin{array}{l}\text { - Maytenus floribunda Reissek. } \\
\text { - Peritassa campestris (Cambess.) A.C. Sm. } \\
\text { - Salacia crassifolia (Mart. ex Schult.) G.Don. }\end{array}$ \\
\hline CHLORANTHACEAE & - Hedyosmum brasiliense Mart. ex Miq. \\
\hline CHRYSOBALANACEAE & $\begin{array}{l}\text { - Hirtella L. } \\
\text { - Hirtella glandulosa Spreng. } \\
\text { - Hirtella gracilipes (Hook.f.) Prance } \\
\text { - Hirtella martiana Hook. f. } \\
\text { - Licania Aubl. }\end{array}$ \\
\hline CONNARACEAE & $\begin{array}{l}\text { - Connarus L. } \\
\text { - Rourea Aubl. } \\
\text { - Rourea chrysomalla Glaz. ex G.Schellenb. }\end{array}$ \\
\hline CONVOLVULACEAE & $\begin{array}{l}\text { - Evolvulus L. } \\
\text { - Ipomoea coccinea L. } \\
\text { - Jacquemontia tamnifolia (L.) Griseb. } \\
\text { - Merremia digitata (Spreng.). Hallier f. } \\
\text { - Merremia tomentosa (Choisy) Hallier f. }\end{array}$ \\
\hline
\end{tabular}


QUADRO 2. Cont. Lista de famílias e espécies doadas ao Herbário Prof. Aluísio Bittencourt, do Centro de Estudos Superiores de Caxias/CESC-UEMA.

\begin{tabular}{|c|c|}
\hline COMBRETACEAE & $\begin{array}{l}\text { - Combretum Loefl. } \\
\text { - Combretum vernicosum Rusby } \\
\text { - Terminalia L. } \\
\text { - Terminalia fagifolia Mart. }\end{array}$ \\
\hline CYPERACEAE & - Cyperus L. \\
\hline DILLENIACEAE & $\begin{array}{l}\text { - Davilla elliptica A. St.-Hil. } \\
\text { - Davilla grandiflora A.St.-Hil. }\end{array}$ \\
\hline DIOSCOREACEAE & - Dioscorea L. \\
\hline EBENACEAE & $\begin{array}{l}\text { - Diospyros hispida A.DC. } \\
\text { - Diospyros sericea A. DC. }\end{array}$ \\
\hline ERICACEAE & - Leucothoe chorantha (Cham.) G.Don. \\
\hline ERIOCAULACEAE & $\begin{array}{l}\text { - Eriocaulon L. } \\
\text { - Paepalanthus Mart. }\end{array}$ \\
\hline ERYTROXYLACEAE & $\begin{array}{l}\text { - Erythroxylum P.Browne } \\
\text { - Erythroxylum columbinum Mart. } \\
\text { - Erythroxylum Pelleterianum A. St.-Hil. }\end{array}$ \\
\hline EUPHORBIACEAE & $\begin{array}{l}\text { - Alchornea glandulosa Poepp \& Endl. } \\
\text { - Croton L. } \\
\text { - Croton goyazensis Miill. Arg. } \\
\text { - Maprounea guianensis Aubl. } \\
\text { - Sebastiania Spreng. } \\
\text { - Sebastiania bidentata (Mart. \& Zucc.). Pax }\end{array}$ \\
\hline & $\begin{array}{l}\text { - Abarema cochliacarpos (Gomes) Barneby \& J.W. } \\
\text { Grimes } \\
\text { - Acacia polyphylla DC. } \\
\text { - Aeschynomene L. } \\
\text { - Aeschynomene paniculata Willd. ex Vogel } \\
\text { - Andira humilis Mart. Ex Benth. } \\
\text { - Camptosema spectabile (Tul.). Burkat }\end{array}$ \\
\hline
\end{tabular}


QUADRO 2. Cont. Lista de famílias e espécies doadas ao Herbário Prof. Aluísio Bittencourt, do Centro de Estudos Superiores de Caxias/CESC-UEMA.

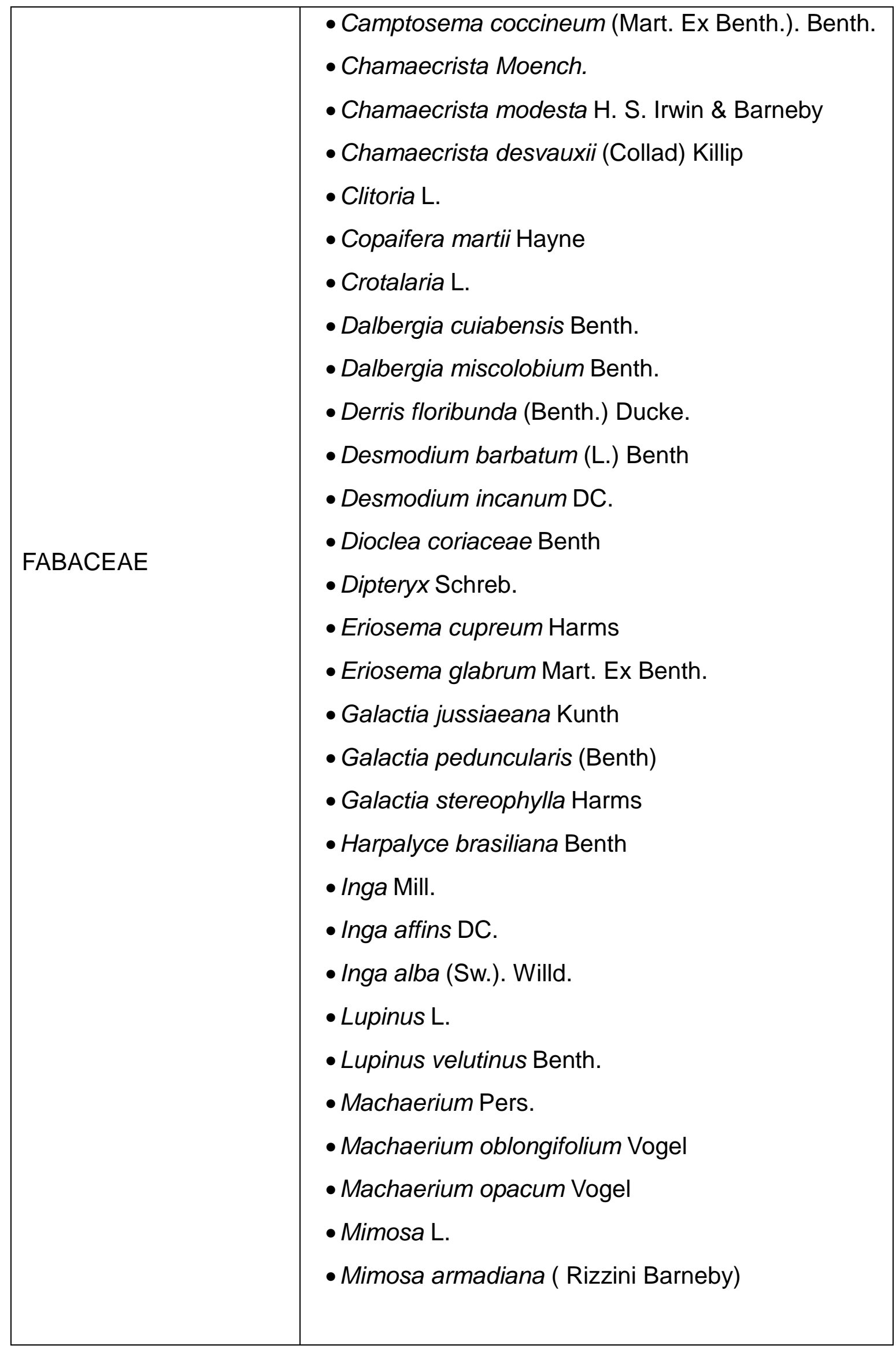


QUADRO 2. Cont. Lista de famílias e espécies doadas ao Herbário Prof. Aluísio Bittencourt, do Centro de Estudos Superiores de Caxias/CESC-UEMA.

\begin{tabular}{|c|c|}
\hline & $\begin{array}{l}\text { - Mimosa diplotricha (Wright ex Sauvale) } \\
\text { - Mimosa nuda (Benth) Barneby } \\
\text { - Mucuna urens (L.) Medik. } \\
\text { - Periandra heterophylla Benth } \\
\text { - Periandra mediterrânea (Vell) Taub } \\
\text { - Plathymenia reticulata Benth. } \\
\text { - Poiretia cariifolia Vogel } \\
\text { - Rhynchosia pyramidalis (Lam.) Urb. } \\
\text { - Senna Mill } \\
\text { - Senna cana (Nees \&Mart) } \\
\text { - Senna rugosa (G. Don) H. S. Irwin \& Barneby } \\
\text { - Stryphnodendron barbatulum Rizzini \& Heringer } \\
\text { - Stylosanthes Sw. } \\
\text { - Stylosanthes gracilis Kunth } \\
\text { - Stylosanthes guianensis (Aubl.) Sw. } \\
\text { - Vigna peduncularis (Kunth) Fawc.\& Rendle } \\
\text { - Zornia latifolia Sm. }\end{array}$ \\
\hline HYMENOPHYLLACEAE & - Trichomanes L. \\
\hline IRIDACEAE & - Sisyrinchium vaginatum Spreng. \\
\hline KRAMERIACEAE & - Krameria tomentosa A. St.-Hil. \\
\hline LAMIACEAE & $\begin{array}{l}\text { - Amasonia L.f } \\
\text { - Hyptis Jacq. }\end{array}$ \\
\hline LAURACEAE & $\begin{array}{l}\text { - Mezilaurus crassiramea (Meisn.). Taub. ex Mez } \\
\text { - Ocotea Aubl. } \\
\text { - Ocotea macropoda (Kunth) Mez }\end{array}$ \\
\hline LOGANIACEAE & - Pseudospigelia W. Klett \\
\hline LORANTHACEAE & $\begin{array}{l}\text { - Phthirusa Mart. } \\
\text { - Phthirusa ovata (Pohl ex DC.). Eichler } \\
\text { - Psittacanthus robustos Mart. } \\
\text { - Strutanthus Mart. }\end{array}$ \\
\hline
\end{tabular}


QUADRO 2. Cont. Lista de famílias e espécies doadas ao Herbário Prof. Aluísio Bittencourt, do Centro de Estudos Superiores de Caxias/CESC-UEMA.

\begin{tabular}{|c|c|}
\hline & - Strutanthus flexicaulis Mart. \\
\hline LYTHRACEAE & $\begin{array}{l}\text { - Cuphea P. Browne } \\
\text { - Cuphea flava Spreng. } \\
\text { - Cuphea melvilla Lindl. } \\
\text { - Cuphea sesilifolia Mart. } \\
\text { - Diplusodon Pohl } \\
\text { - Diplusodon helianthemifolius DC. } \\
\text { - Diplusodon hexander DC. } \\
\text { - Diplusodon oblongos Pohl } \\
\text { - Diplusodon ramosissimus Pohl. } \\
\text { - Diplusodon rosmarinifolius A. St.-Hil. } \\
\text { - Diplusodon sessiliflorus Koehne } \\
\text { - Diplusodon virgatus Pohl } \\
\text { - Punica L. }\end{array}$ \\
\hline MAGNOLIACEAE & - Talauma ovata St.-Hil. \\
\hline MALPIGHIACEAE & $\begin{array}{l}\text { - Banisteriopsis C.B.Rob. ex Small } \\
\text { - Banisteriopsis latifolia (A. Juss.) } \\
\text { - Banisteriopsis malifolia (Nees \& Mart.) } \\
\text { - Banisteriopsis stellaris (Griseb.) } \\
\text { - Byrsonima Rich. Ex Kunth } \\
\text { - Byrsonima brasiloba A. Juss. } \\
\text { - Byrsonima crassa Nied. } \\
\text { - Heteropterys affinis A. Juss. } \\
\text { - Mascagnia Bertero } \\
\text { - Tetrapterys sp. Cav. }\end{array}$ \\
\hline MALVACEAE & $\begin{array}{l}\text { - Hibiscos L. } \\
\text { - Luhea Wild. } \\
\text { - Pavonia malacophylla (Link \& Otto) Garcke } \\
\text { - Pavonia rosa-campestris A.St. -Hil. } \\
\text { - Triumfetta L. } \\
\text { - Triumfetta semitriloba Jacq. }\end{array}$ \\
\hline
\end{tabular}


QUADRO 2. Cont. Lista de famílias e espécies doadas ao Herbário Prof. Aluísio Bittencourt, do Centro de Estudos Superiores de Caxias/CESC-UEMA.

\begin{tabular}{|c|c|}
\hline & - Waltheria operculata Rose. \\
\hline MARANTACEAE & $\begin{array}{l}\text { - Calathea G.Mey. } \\
\text { - Calathea capitata (Ruiz \& Pav.) Lindl. } \\
\text { - Maranta pleiostachys K. Schum. }\end{array}$ \\
\hline MELASTOMATACEAE & $\begin{array}{l}\text { - Miconia Ruiz \& Pav. } \\
\text { - Miconia burchellii Triana } \\
\text { - Miconia polyandra Gardner } \\
\text { - Microlicia fulva (Spreng.) Cham. } \\
\text { - Rhynchanthera grandiflora (Aubl.) } \\
\text { - Rhynchanthera rostrata DC. }\end{array}$ \\
\hline MELIACEAE & $\begin{array}{l}\text { - Cabralea canjerana (Vell.) Mart. } \\
\text { - Guarea guidonia (L.) Sleumer } \\
\text { - Guarea kunthiana A. Juss. } \\
\text { - Trichilia catigua A.Juss. } \\
\text { - Trichilia elegans A. Juss. } \\
\text { - Trichilia pallida Sw. }\end{array}$ \\
\hline MENISPERMACEAE & - Cissampelos ovalifolia DC. \\
\hline MORACEAE & $\begin{array}{l}\text { - Dorstenia L. } \\
\text { - Ficus L. } \\
\text { - Ficus guaranitica Chotdat. }\end{array}$ \\
\hline MYRTACEAE & $\begin{array}{l}\text { - Blepharocalyx Salcifolius (Kunth) O. Berg } \\
\text { - Campomanesia sessiliflora (O. Berg) Mattos } \\
\text { - Campomanesia velutina (Cambess.) } \\
\text { - Eugenia bracteata Vell. } \\
\text { - Eugenia involucrata DC. } \\
\text { - Eugenia klotzschiana O. Berg } \\
\text { - Eugenia punicifolia (Kunth) DC. } \\
\text { - Eugenia stenosepala Kiaersk Cambess. } \\
\text {-Eugenia uruguayensis Psidium sp L. } \\
\text { - Gomidesia O. Berg. }\end{array}$ \\
\hline
\end{tabular}


QUADRO 2. Cont. Lista de famílias e espécies doadas ao Herbário Prof. Aluísio Bittencourt, do Centro de Estudos Superiores de Caxias/CESC-UEMA.

\begin{tabular}{|c|c|}
\hline & $\begin{array}{l}\text { - Gomidesia lindeniana Berg } \\
\text { - Myrcia DC. } \\
\text { - Myrcia cordiifolia DC. } \\
\text { - Myrcia goyazensis Cambess. } \\
\text { - Myrcia rorida (O. Berg.) Kiaersk } \\
\text { - Myrcia rostrata DC. } \\
\text { - Myrcia sellowiana O. Berg. } \\
\text { - Myrcia tomentosa (Aubl.) DC. } \\
\text { - Myrcia torta DC. } \\
\text { - Psidium L. } \\
\text { - Psidium myrsinoides O. Berg } \\
\text { - Psidium rufum Mart. Ex DC. } \\
\text { - Siphoneugena densiflora O. Berg }\end{array}$ \\
\hline NYCTAGINACEAE & - Guapira graciliflora (Mart. Ex Schmidt) Lundell \\
\hline OCHNACEAE & $\begin{array}{l}\text { - Ouratea Aubl. } \\
\text { - Ouratea acuminata (A.DC.) Engl. } \\
\text { - Ouratea castaneifolia (DC.) Engl. } \\
\text { - Ouratea floribunda Engl. } \\
\text { - Ouratea hexasperma (A.St.-Hil.) Baill } \\
\text { - Sauvagesia racemosa A.St.-Hil. }\end{array}$ \\
\hline OLACACEAE & - Heisteria Jaq. \\
\hline ONAGRACEAE & $\begin{array}{l}\text { - Ludwigia L. } \\
\text { - Ludwigia nervosa (Poir) H. Hara } \\
\text { - Ludwigia tomentosa (Cambess.) H. Hara }\end{array}$ \\
\hline ORCHIDACEAE & $\begin{array}{l}\text { - Epidendrum elongatum Jacq. } \\
\text { - Epidendrum secundum Jacq. }\end{array}$ \\
\hline OROBANCHACEAE & - $\quad$ Esterhazya Splendida JC Mikan \\
\hline PHYLLANTHACEAE & •Richeria grandis Vahl \\
\hline PIPERACEAE & $\begin{array}{l}\text { - Peperomia gardneriana Miq. } \\
\text { - Piper L. }\end{array}$ \\
\hline
\end{tabular}


QUADRO 2. Cont. Lista de famílias e espécies doadas ao Herbário Prof. Aluísio Bittencourt, do Centro de Estudos Superiores de Caxias/CESC-UEMA.

\begin{tabular}{|c|c|}
\hline & - Piper arboreum Aubl. \\
\hline PLUMBAGINACEAE & - Plumbago L. \\
\hline POLYGALACEAE & - Securidaca L. \\
\hline POLYGONACEAE & $\begin{array}{l}\text { - Coccoloba P.Browne } \\
\text { - Coccoloba brasiliensis Nees \& Mart. } \\
\text { - Coccoloba mollis Casar. } \\
\text { - Triplaris Loefl. }\end{array}$ \\
\hline POACEAE & $\begin{array}{l}\text { - Andropogon L. } \\
\text { - Andropogon selloanus (Hack.) Hack. } \\
\text { - Arislida longifólia Trin. } \\
\text { - Axonopus P. Beauv. } \\
\text { - Axonopus eminens (Nees) G.A.Black } \\
\text { - Axonopus marginatus (Trin.) Chase } \\
\text { - Digitaria ciliares (Retz.) Koeler } \\
\text { - Echinolaena inflexa (Poir.) Chase } \\
\text { - Eleusine indica (L.) Gaertn. } \\
\text { - Eriochrysis cayennensis P. Beauv. } \\
\text { - Lasiacis (Griseb.) Hitchc. } \\
\text { - Leptocoryphium Nees } \\
\text { - Mesosetum curtifolium Swallen. } \\
\text { - Panicum cervicatum Chase } \\
\text { - Panicum ligulare Nees ex Trin. } \\
\text { - Panicum millegrama Poir. } \\
\text { - Panicum pilosum Sw. } \\
\text { - Panicum sellowii Nees } \\
\text { - Paspalum L. } \\
\text { - Paspalum ellipticum Döll } \\
\text { - Paspalum gardnerianum Nees } \\
\text { - Paspalum glabrum Poir. } \\
\text { - Paspalum lanciflorum Nees ex Steud. }\end{array}$ \\
\hline
\end{tabular}


QUADRO 2. Cont. Lista de famílias e espécies doadas ao Herbário Prof. Aluísio Bittencourt, do Centro de Estudos Superiores de Caxias/CESC-UEMA.

\begin{tabular}{|c|c|}
\hline & $\begin{array}{l}\text { - Paspalum lineare Trin. } \\
\text { - Paspalum multicaule Poir. } \\
\text { - Raddiella esenbeckii (Steud.) C.E. Calderón \& } \\
\text { Soderstr. } \\
\text { - Schizachyrium hirtiflorum Nees } \\
\text { - Schizachyrium microstachyum (Desv. ex Ham.) } \\
\text { Roseng., B.R. Arrill. \& Izag. } \\
\text { - Schizachyrium scoparium (Michx.) Nash } \\
\text { - Streptachne R. Br. } \\
\text { - Thrasya petrosa (Trin.) Chase } \\
\text { - Trachypogon spicatus (L. f.) Kuntze }\end{array}$ \\
\hline PODOCARPACEAE & - Podocarpus brasiliensis Laubenf. \\
\hline PONTEDERIACEAE & - Pontederia paniculata Spreng. \\
\hline PRIMULACEAE & $\begin{array}{l}\text { - Cibianthus Mart. } \\
\text { - Cibianthus detergens Mart. } \\
\text { - Rapanea guianensis Aubl. }\end{array}$ \\
\hline PTERIDACEAE & $\begin{array}{l}\text { - Adiantum L. } \\
\text { - Adiantum pulerulentum L. } \\
\text { - Adiantum tetraphyllum Hook. }\end{array}$ \\
\hline RANUNCULACEAE & - Clematis dioica L. \\
\hline RAPATACEAE & - Cephalostemon R.H.Schomb \\
\hline RHAMNACEAE & - Rhamnus L. \\
\hline ROSACEAE & - Parinari Aubl. \\
\hline RUBIACEAE & $\begin{array}{l}\text { - Augusta longifolia (Spreng.) Rehder } \\
\text { - Borreria G. Mey. } \\
\text { - Borreria verticillata (L.) G. Mey. } \\
\text { - Coccocypseium P. Browne } \\
\text { - Coussarea platyphylla Müll. Arg. } \\
\text { - Ixora L. } \\
\text { - Palicourea Aubl. } \\
\text { - Palicourea rígida Kunth. }\end{array}$ \\
\hline
\end{tabular}


QUADRO 2. Cont. Lista de famílias e espécies doadas ao Herbário Prof. Aluísio Bittencourt, do Centro de Estudos Superiores de Caxias/CESC-UEMA.

\begin{tabular}{|c|c|}
\hline & $\begin{array}{l}\text { - Psychothria sp. L. } \\
\text { - Sabicea brasiliensis Wernham. } \\
\text { - Sphinctanthus microphyllus K. Schum. } \\
\text { - Tocoyena Aubl. } \\
\text { - Tocoyena formosa (Cham. \& Schltdl.) K. Schum. }\end{array}$ \\
\hline RUTACEAE & $\begin{array}{l}\text { - Esembeckia J. Barbosa Rodrigue } \\
\text { - Zanthoxylum riedelianum Engl. }\end{array}$ \\
\hline SALICACEAE & $\begin{array}{l}\text { - Casearia Jacq. } \\
\text { - Casearia arborea (Rich.) Urb. } \\
\text { - Casearia grandiflora Cambess. }\end{array}$ \\
\hline SANTALACEAE & $\begin{array}{l}\text { - Phoradendrom Nutt. } \\
\text { - Phoradendrom bacthyoryctum Eichler } \\
\text { - Phoradendrom crassifolium (Pohl ex DC.) Eichler }\end{array}$ \\
\hline SAPINDACEAE & $\begin{array}{l}\text { - Allophylus L. } \\
\text { - Allophylus edulis (A. St.-Hil., A. Juss. \& Cambess.) } \\
\text { Hieron. ex Niederl; } \\
\text { - Cardiospermum L. } \\
\text { - Cupania L. } \\
\text { - Matayba guianensis Aubl } \\
\text { - Salvia brevipes Benth. } \\
\text { - Serjania Mill. } \\
\text { - Toulicia Aubl }\end{array}$ \\
\hline SAPOTACEAE & - Chrysophyllum L. \\
\hline SIMAROUBACEAE & - Simaba blanchetii Turcz. \\
\hline SIPARUNACEAE & $\begin{array}{l}\text { - Siparuna Aubl. } \\
\text { - Siparuna guianensis Aubl. }\end{array}$ \\
\hline SOLANACEAE & $\begin{array}{l}\text { - Brunfelsia brasiliensis (Spreng.) L.B.Sm. \& Downs } \\
\text { - Cestrum L. } \\
\text { - Cestrum macrophyllum Salzm. ex Dunal. } \\
\text { - Cestrum megalophyllum Dunal }\end{array}$ \\
\hline
\end{tabular}


QUADRO 2. Cont. Lista de famílias e espécies doadas ao Herbário Prof. Aluísio Bittencourt, do Centro de Estudos Superiores de Caxias/CESC-UEMA.

\begin{tabular}{|c|c|}
\hline & $\begin{array}{l}\text { - Lycopersicon Mill. } \\
\text { - Solanum L. } \\
\text { - Solanum granulosoleprosum Dunal } \\
\text { - Solanum lycocarpum A.St.-Hil. } \\
\text { - Solanum subumbellatum Vell. }\end{array}$ \\
\hline SYMPLOCACEAE & - Symplocos nitens (Pohl.) Benth. \\
\hline STYRACACEAE & $\begin{array}{l}\text { - Styrax L. } \\
\text { - Styrax ambiguous Seub. } \\
\text { - Styrax parviflorus Merr. }\end{array}$ \\
\hline TURNERACEAE & - Turnera lamiifolia Cambess. \\
\hline URTICACEAE & $\begin{array}{l}\text { - Fleurya aestuans (L.) Gaudich. Ex Miq. } \\
\text { - Urera baccífera (L.) Gaudich. }\end{array}$ \\
\hline VELLOZIACEAE & - Vellozia squamata Pohl. \\
\hline VERBENACEAE & $\begin{array}{l}\text { - Lantana achyranthifolia Desf. } \\
\text { - Lantana hypoleuca Briq. } \\
\text { - Lippia glandulosae Schauer. } \\
\text { - Lippia lupulina Cham. } \\
\text { - Stachyt schauerii Moldenke }\end{array}$ \\
\hline VIOLACEAE & $\begin{array}{l}\text { - Hybanthus atropurpureus (A.St.-Hil.) Taub. } \\
\text { - Rinorea Aubl. }\end{array}$ \\
\hline VITACEAE & $\begin{array}{l}\text { - Cissus L. } \\
\text { - Cissus salutaris Kunth. } \\
\text { - Cissus simsiana Schult. \& Schult.f. }\end{array}$ \\
\hline VOCHYSIACEAE & $\begin{array}{l}\text { - Callisthene Mart. } \\
\text { - Callisthene major Mart. } \\
\text { - Qualea Aubl. } \\
\text { - Qualea dichotoma (Mart.) Quente. } \\
\text { - Qualea grandiflora Mart. } \\
\text { - Qualea multiflora Mart. } \\
\text { - Qualea parviflora Mart. }\end{array}$ \\
\hline
\end{tabular}


QUADRO 2. Cont. Lista de famílias e espécies doadas ao Herbário Prof. Aluísio Bittencourt, do Centro de Estudos Superiores de Caxias/CESC-UEMA.

\begin{tabular}{|l|l|}
\hline & $\bullet$ Qualea wiittrockii Malme \\
& $\bullet$ Vochysia Aubl. \\
& $\bullet$ Vochysia elliptica Mart. \\
& $\bullet$ Vochysia herbacea Pohl \\
& $\bullet$ Vochysia pyramidalis Mart. \\
& $\bullet$ Vochysia rufa Mart. \\
& $\bullet$ Vochysia thyrsoidea Pohl \\
\hline XYRIDACEAE & $\bullet$ Xyris asperula Mart. \\
\hline
\end{tabular}

Tabela 1. Distribuição do número de gêneros, de espécies e espécimes doados ao Herbário Prof. Aluísio Bittencourt por família.

\begin{tabular}{c|c|c|c}
\hline Família & $\begin{array}{c}\text { Número de } \\
\text { Gênero }\end{array}$ & $\begin{array}{c}\text { Número de } \\
\text { Espécie }\end{array}$ & $\begin{array}{c}\text { Número de } \\
\text { Espécimes }\end{array}$ \\
\hline ACANTHACEAE & 4 & 5 & 6 \\
\hline ALSTROMERIACEAE & 1 & 1 & 1 \\
\hline AMARANTHACEAE & 1 & 2 & 2 \\
\hline AMARYLLIDACEAE & 1 & 1 & 1 \\
\hline ANEMIACEAE & 1 & & 3 \\
\hline ANNONACEAE & 4 & 6 & 7 \\
\hline APOCYNACEAE & 1 & 1 & 1 \\
\hline ARACEAE & 2 & 2 & 2 \\
\hline ARALIACEAE & 2 & 3 & 5 \\
\hline ARECACEAE & 1 & 1 & 2 \\
\hline ARISTOLOCHIACEAE & 1 & 2 & 2 \\
\hline ASTERACEAE & 10 & 24 & 44 \\
\hline BIGNONIACEAE & 3 & 5 & 7 \\
\hline BIXACEAE & 2 & 3 & 1 \\
\hline BLECHNACEAE & 1 & 1 & 5 \\
\hline BORAGINACEAE & 2 & 4 & 4 \\
\hline BURSERACEAE & 3 & 4 & 1 \\
\hline CAMPANULACEAE & 1 & 1 & 1 \\
\hline CELASTRACEAE & 3 & 3 & 8 \\
\hline CHLORANTHACEAE & 1 & 1 & 1 \\
\hline CHRYSOBALANACEAE & 2 & 5 & 3
\end{tabular}


Tabela 1. Cont. Distribuição do número de gêneros, de espécies e espécimes doados ao Herbário Prof. Aluísio Bittencourt por família.

\begin{tabular}{|c|c|c|c|}
\hline CONVOLVULACEAE & 4 & 5 & 5 \\
\hline COMBRETACEAE & 2 & 4 & 5 \\
\hline CYPERACEAE & 1 & 1 & 1 \\
\hline DILLENIACEAE & 1 & 2 & 3 \\
\hline DIOSCOREACEAE & 1 & 1 & 3 \\
\hline EBENACEAE & 1 & 2 & 2 \\
\hline ERICACEAE & 1 & 1 & 1 \\
\hline ERIOCAULACEAE & 2 & 2 & 2 \\
\hline ERYTROXYLACEAE & 1 & 3 & 4 \\
\hline EUPHORBIACEAE & 4 & 6 & 12 \\
\hline FABACEAE & 30 & 53 & 63 \\
\hline HYMENOPHYLLACEAE & 1 & 1 & 1 \\
\hline IRIDACEAE & 1 & 1 & 1 \\
\hline KRAMERIACEAE & 1 & 1 & 1 \\
\hline LAMIACEAE & 2 & 2 & 2 \\
\hline LAURACEAE & 2 & 3 & 4 \\
\hline LOGANIACEAE & 1 & 1 & 1 \\
\hline LORANTHACEAE & 3 & 5 & 7 \\
\hline LYTHRACEAE & 3 & 13 & 45 \\
\hline MAGNOLIACEAE & 1 & 1 & 1 \\
\hline MALPIGHIACEAE & 5 & 10 & 12 \\
\hline MALVACEAE & 5 & 7 & 12 \\
\hline MARANTACEAE & 2 & 3 & 5 \\
\hline MELASTOMATACEAE & 2 & 6 & 7 \\
\hline MELIACEAE & 3 & 6 & 9 \\
\hline MENISPERMACEAE & 1 & 1 & 1 \\
\hline MORACEAE & 2 & 3 & 4 \\
\hline MYRTACEAE & 7 & 23 & 35 \\
\hline NYCTAGINACEAE & 1 & 1 & 2 \\
\hline OCHNACEAE & 2 & 6 & 9 \\
\hline OLACACEAE & 1 & 1 & 1 \\
\hline ONAGRACEAE & 1 & 3 & 4 \\
\hline ORCHIDACEAE & 1 & 2 & 2 \\
\hline OROBANCHACEAE & 1 & 1 & 1 \\
\hline PHYLLANTHACEAE & 1 & 1 & 1 \\
\hline PIPERACEAE & 2 & 3 & 6 \\
\hline PLUMBAGINACEAE & 1 & 1 & 1 \\
\hline POLYGALACEAE & 1 & 1 & 2 \\
\hline POLYGONACEAE & 2 & 4 & 5 \\
\hline POACEAE & 17 & 32 & 57 \\
\hline PODOCARPACEAE & 1 & 1 & 1 \\
\hline PONTEDERIACEAE & 1 & 1 & 2 \\
\hline PRIMULACEAE & 2 & 3 & 4 \\
\hline
\end{tabular}


Tabela 1. Cont. Distribuição do número de gêneros, de espécies e espécimes doados ao Herbário Prof. Aluísio Bittencourt por família.

\begin{tabular}{l|c|c|c} 
PTERIDACEAE & 1 & 3 & 3 \\
\hline RANUNCULACEAE & 1 & 1 & 1 \\
\hline RAPATACEAE & 1 & 1 & 1 \\
\hline RHAMNACEAE & 1 & 1 & 1 \\
\hline ROSACEAE & 1 & 1 & 2 \\
\hline RUBIACEAE & 10 & 13 & 3 \\
\hline RUTACEAE & 2 & 2 & 3 \\
\hline SALICACEAE & 1 & 3 & 14 \\
\hline SANTALACEAE & 1 & 3 & 1 \\
\hline SAPINDACEAE & 7 & 8 & 1 \\
\hline SAPOTACEAE & 1 & 1 & 25 \\
\hline SIMAROUBACEAE & 1 & 1 & 2 \\
\hline SIPARUNACEAE & 1 & 2 & 3 \\
\hline SOLANACEAE & 4 & 9 & 2 \\
\hline SYMPLOCACEAE & 1 & 2 & 3 \\
\hline STYRACEAE & 1 & 3 & 1 \\
\hline TURNERACEAE & 1 & 1 & 5 \\
\hline URTICACEAE & 2 & 2 & 2 \\
\hline VELLOZIACEAE & 1 & 1 & 5 \\
\hline VERBENACEAE & 3 & 5 & 1 \\
\hline VIOLACEAE & 2 & 2 & 54 \\
\hline VITACEAE & 1 & 3 & 645 \\
\hline VOCHYSIACEAE & 3 & 14 & \\
\hline XYRIDACEAE & 1 & 1 & ---- \\
\hline S/ID. & ---- & 391 & \\
\hline TOTAL: & 223 & & 23 \\
\hline & & & \\
\hline
\end{tabular}

Os dados obtidos revelam um grande contingente de espécimes doados depositadas no Herbário, representando aproximadamente 16\% do número total de espécies, observando-se também um expressivo número de famílias, gêneros e espécies encontradas entre os exemplares analisados. Dentre as famílias acervadas no Herbário, 15 contém entre seus representantes no acervo apenas espécimes doados, sendo as seguintes famílias botânicas: Symplocaceae, Styracaceae, Urticaceae, Violaceae, Ranunculaceae, Rapataceae, Polygalaceae, Pontederiaceae, Podocarpaceae,Orobanchaceae, Siparunaceae, Hymenophyllaceae, Dioscoreaceae, Bixaceae e Anemiaceae, isso reflete a necessidade da realização de novas coletas para se ter acesso ao conhecimento da flora de modo geral.

As coleções científicas têm papel fundamental na realização do registro da biodiversidade. De acordo com RODRIGUES et al., (1981) o Herbário do INPA tem mantido intenso intercâmbio com varias instituições nacionais e internacionais, permutando e recebendo materiais botânicos para estudos, como também vem recebendo visitas a fim de conhecer suas coleções e obter dados sobre plantas nativas. A coleção do Herbário do INPA vem apresentando uma importante fonte de consulta para os taxonomistas, fitogeógrafos e outros estudiosos. 
Com o advento tecnológico os Herbários perceberam a necessidade de informatizar suas coleções com o objetivo de divulgar de modo rápido e eficiente às informações. Neste sentido, o Herbário MAR vem trabalhando para disponibilizar os dados das amostras vegetais no banco de dados do specieLink, possibilitando a divulgação da flora do Estado (SILVA et al., 2013).

Recentemente os Herbários começaram a disponibilizar fotos e informações sobre suas coleções on-line. Esta prática reduziu o envio de material somente para aqueles casos em que é estritamente necessário (SCHMULL et al., 2005). A informatização também pode auxiliar o intercambio de modo a agilizar este processo, podendo diminuir o uso dos correios, diminuindo danificação do material ou a perda dos pacotes durante o envio, pois este processo é demorado e pouco seguro como diz PEIXOTO et al., (2006).

O Herbário do Campus de Pesquisa Museu Paraense Emílio Goeldi, é um dos Herbários que está em processo de informatização, onde prevê disponibilizar as imagens para uma consulta rápida e possibilitar as comparações entre os registros de diferentes Herbários (MATOS, 2013).

Desse modo, o incremento dos Herbários com as informações vem enriquecendo as coleções botânicas, de modo a disponibilizar dados para estudos sobre a biodiversidade da flora no Brasil como também de outros países.

\section{CONCLUSÃO}

Os resultados obtidos na presente pesquisa refletem a eficiência deste tipo de atividade, uma vez que além da abrangência de diversas áreas, obteve-se também um número bastante representativo de espécimes doados ao Herbário do CESC/UEMA, sendo quantificados 645 espécimes, distribuídos em 90 famílias, 223 gêneros e 391 espécies, um número bastante significativo.

Dentre o total de espécimes, quatro são provenientes de instituições estrangeiras, sendo as demais oriundas de instituições nacionais, com destaque 0 Distrito Federal. Das famílias encontradas as famílias Fabaceae, Poaceae, Asteraceae e Myrtaceae, foram as que apresentaram um maior quantitativo de espécimes doados.

O intercâmbio entre Herbários já faz parte da rotina de grande parte dos Herbários, isso por ser uma atividade que propicia inúmeras vantagens. Uma dessas vantagens é que com a inserção de novos espécimes as coleções, a amplitude e abrangência das coleções botânicas têm se estendido a cada dia, enriquecendo as mesmas e aperfeiçoando o processo de identificação. Isso contribui para o conhecimento da flora de modo geral, bem como incentivo à realização de novas coletas botânicas, tanto para a implementação dos Herbários, como para o conhecimento e divulgação da flora local.

\section{REFERÊNCIAS}

BARBOSA, M. R. V.; VIEIRA, A. O. 2005. Coleções de plantas vasculares: Diagnóstico, desafios e estratégias. Disponível em: (www.cria.org.br/cgee/col). Acesso em: 07/01/2014.

HERBÁRIO ICN. Instituto de Biociências, Rio Grande do Sul, 21 de jun. 2011. Disponível em: <http:/www.ufrgs.br/biociências/Acesso em: 09 Fev. 2014. 
LISTA de espécies da flora do Brasil. Jardim Botânico do Rio de Janeiro, Rio de Janeiro, 03 Fev. 2014. Disponível em: <http://floradobrasil.jbri.gov.br/>. Acesso em: 03 Fev. 2014.

MACHADO, S. R.; BARBOSA, S. B. Manual de procedimentos - Herbário BOTU. Botucatu, São Paulo.18 pg. 2010.

MANIA, L. F.; ASSIS, M. A.; Processo de informatização do Herbário Rioclarense (HRCB) da Universidade Estadual Paulista (UNESP), Campus de Rio Claro, SP, e sua inclusão num sistema de rede. Rev. Ciênc. Ext. v.4, n.1, p.8, 2008.

MATOS, J. Informatização e reconhecimento. Museu Goeldi. Pará, 24 de jan. 2013. Disponível em: <http://www.museu-goeldi.br/portal/content/museu-goeldiretoma >>. Acesso em: 11/02/2014.

PEIXOTO, A. L; BARBOSA, M. R. V; CANHOS, D. A. L \& MAIA, L. C. Coleções Botânicas: objetos e dados para a ciência. Cultura Material e Patrimônio de C\&T: 315-326, 2009.

PEIXOTO, A. L.; MORIM, M. P. Coleções botânicas: documentação da biodiversidade brasileira. Ciência \& Cultura, n. 55, v.3, p.21-48. 2003.

PEIXOTO, A. L. BARBOSA, M. R. V; CANHOS, D. A. L \& MAIA, L. C. Diretrizes e estratégias para a modernização de coleções biológicas brasileiras e a consolidação de sistemas integrados de informação sobre biodiversidade, Brasília: MCT/ CGEE, p.145-182. 2006.

RESENDE, M. L. F.; GUIMARÃES, L.L. Inventários da Biodiversidade do Cerrado: Biogeografia de Plantas. Instituto Brasileiro de Geografia e Estatística (IBGE), 2007.

RODRIGUES, W.; SILVA, M. F. O Herbário do INPA. Acta Amazônica, v. 11, n. 4, p. 667-669, 1981.

SCHMULL, M.; HEINRICHS, J.; BAIER, R.; ULLRICH, D.; WAGENITZ, G.; GROTH, H.; HOURTICOLON, S. \& GRADSTEIN, R. The type database at Göttingen (GOET) - a virtual herbarium online. Taxon, v. 54, n. 1, p. 251-254, 2005.

SILVA, A. N. F.; BRITO, D. R. B. ; JUNIOR, E. B. A. Processo de formação do Herbário do Maranhão (MAR), da Universidade Federal do Maranhão. In: 64ํำ CONGRESSO NACIONAL DE BOTÂNICA, 10-15., 2013, Belo Horizonte. Resumos... Belo Horizonte: UFMA, 2013. p.1. Sociedade Botânica do Brasil. Catálogo da Rede de Herbários. Disponível em: <http://www.botanica.org.br/rede_herbarios.php. Acesso em: 07/01/2013.

SOCIEDADE BOTÂNICA DO BRASIL. Catálogo da Rede de Herbários. Disponível em: <http://www.botanica.org.br/rede_herbarios.php. Acesso em: 07/01/2013. 
THIERS, B. [continuamente atualizado]. Índice Herbariorum: Um diretório global de herbários pública e pessoal associado. Herbário Virtual do Jardim Botânico de Nova lorque. http://sweetgum.nybg.org/ih/. Acesso em: 07/01/2014.

Tropicus. Org. Missouri Botanical Garden, Missouri, 07 jan. 2014. Disponível em:<http://www.tropicus. org. Acesso em: 07/01/2014. 


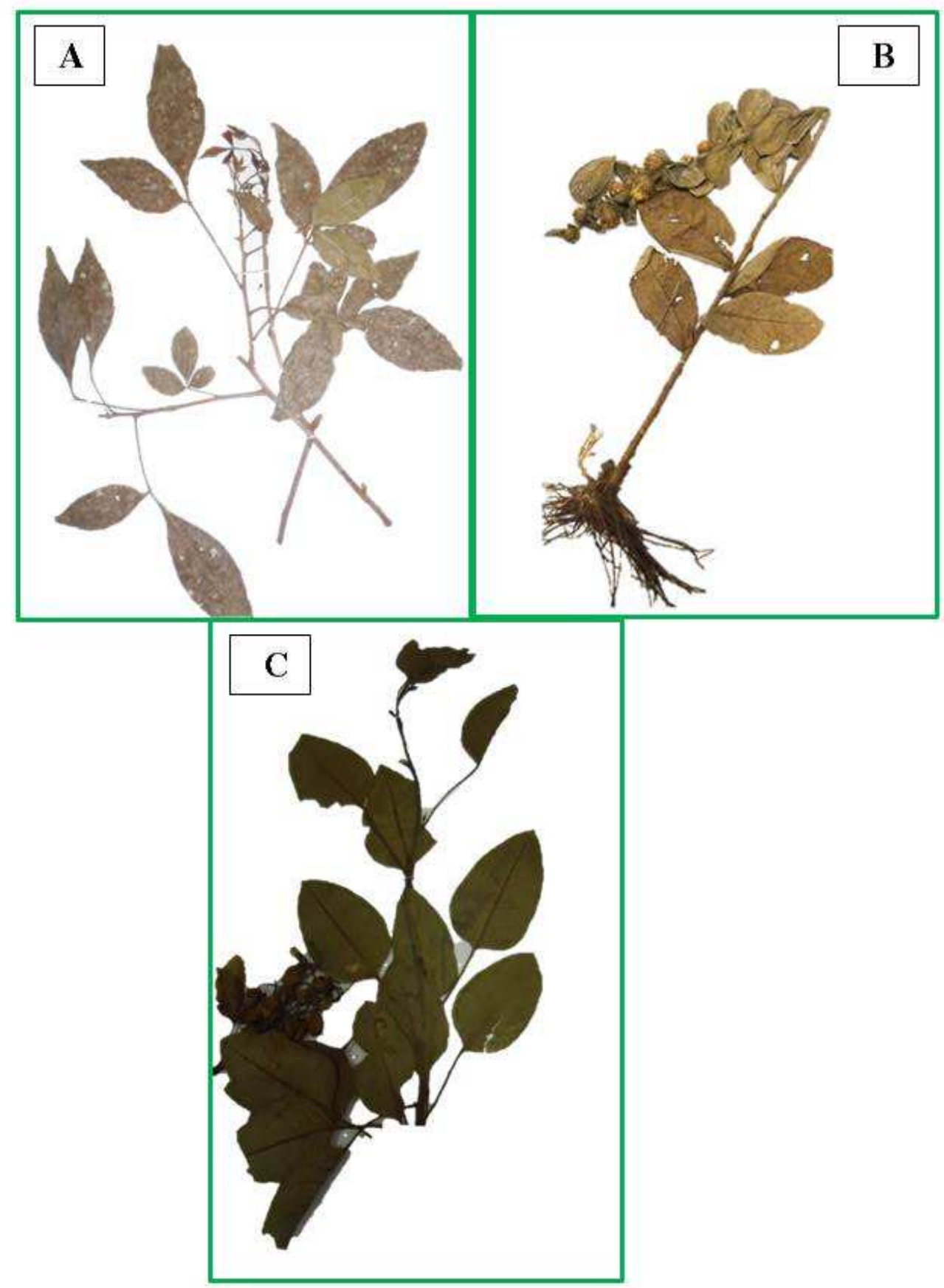

Figura 1: Espécimes doados por Instituições Estrangeiras ao Herbário Professor Aluísio Bittencourt. A) Allophylus edulis (A. St.-Hil., A. Juss. \& Cambess.), (Sapindaceae) doado do Paraguai; B) Vernonia bardanoides Less. (Asteraceae) doado do Paraguai e em C) Brassaia actinophylla Endl.(Araliaceae) doado do Instituto de Botânica Del Nordeste, Argentina. 


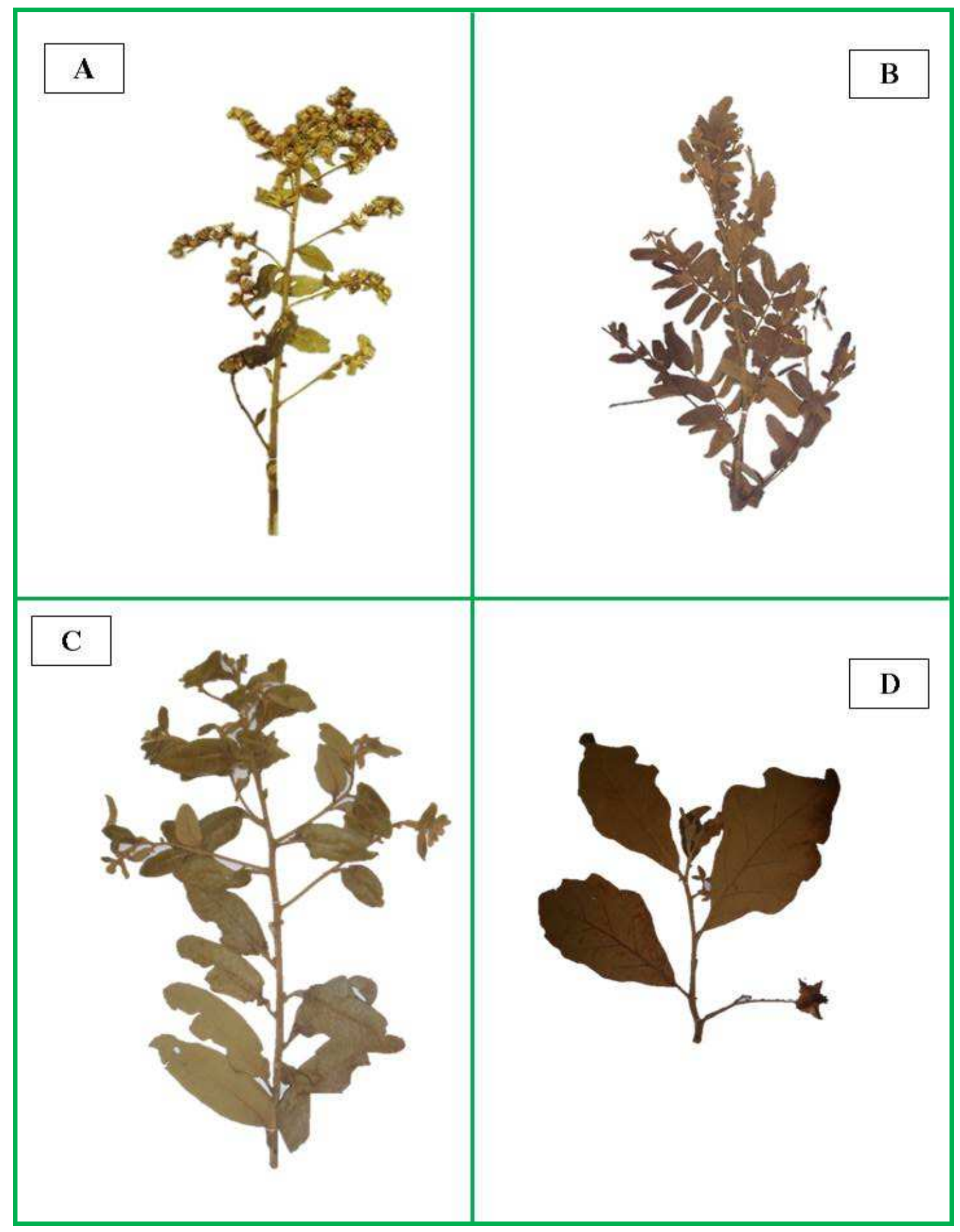

Figura 2: Espécimes doados de diferentes regiões do Brasil ao Herbário Professor Aluísio Bittencourt. A) Vernonia aurea Mart. ex, DC. (Asteraceae) doado do Distrito Federal; B) Harpalyce brasiliana Benth (Fabaceae), doado de Balsas; C) Vernonia ferruginea Less. (Asteraceae) doado de Goiás e em D) Solanum L. (Solanaceae) doado de São Paulo. 


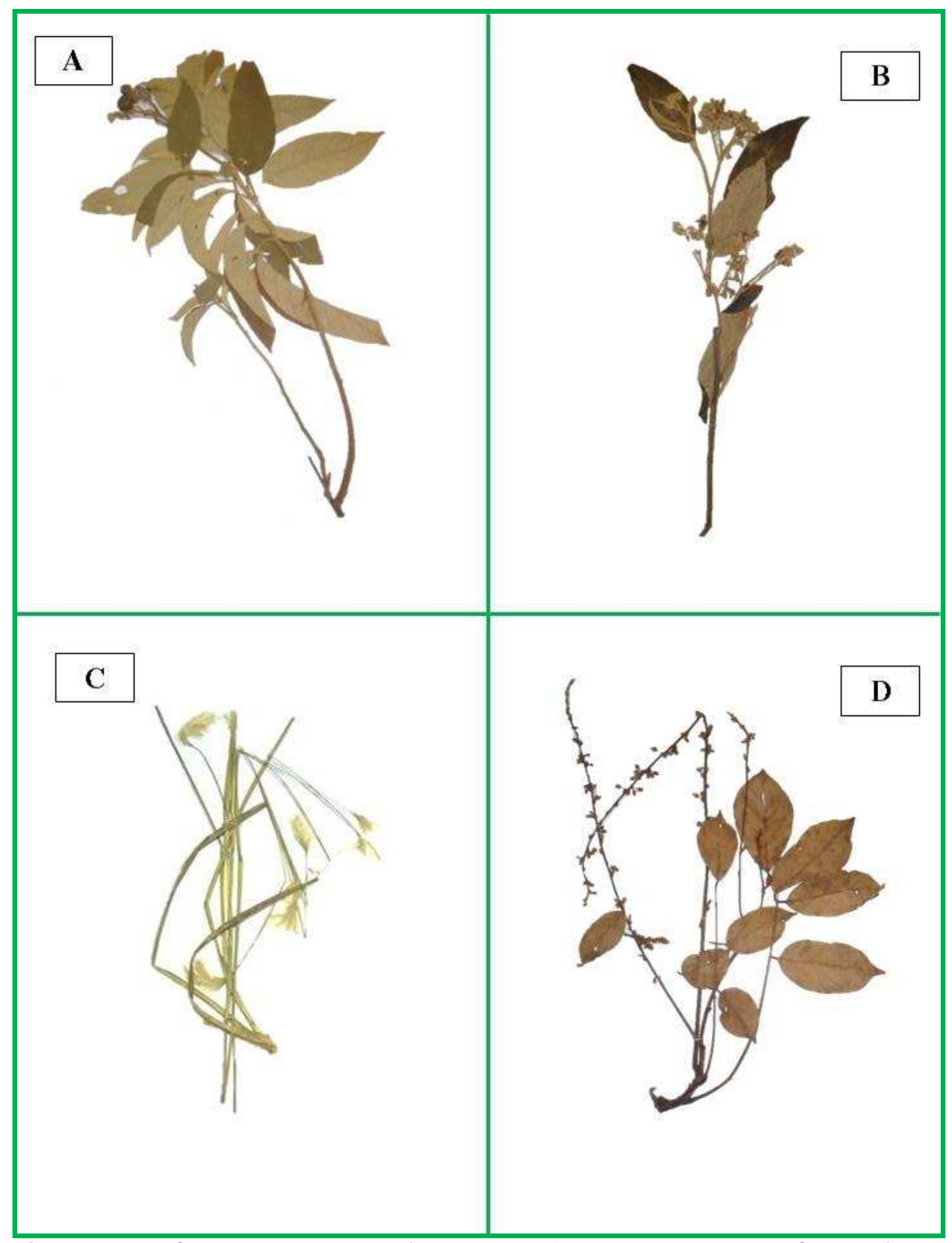

Figura 3: Espécimes doados de diferentes regiões do Brasil ao Herbário Professor Aluísio Bittencourt. A) Solanum granulosoleprosum Dunal (Solanaceae) doado da Bahia; B) Solanum L. (Solanaceae) doado do Mato Grosso do Sul; C) Andropogon L. (Poaceae) doado de Minas Gerais e em D) Derris floribunda (Benth.) Ducke. (Fabaceae) doado do Pará. 


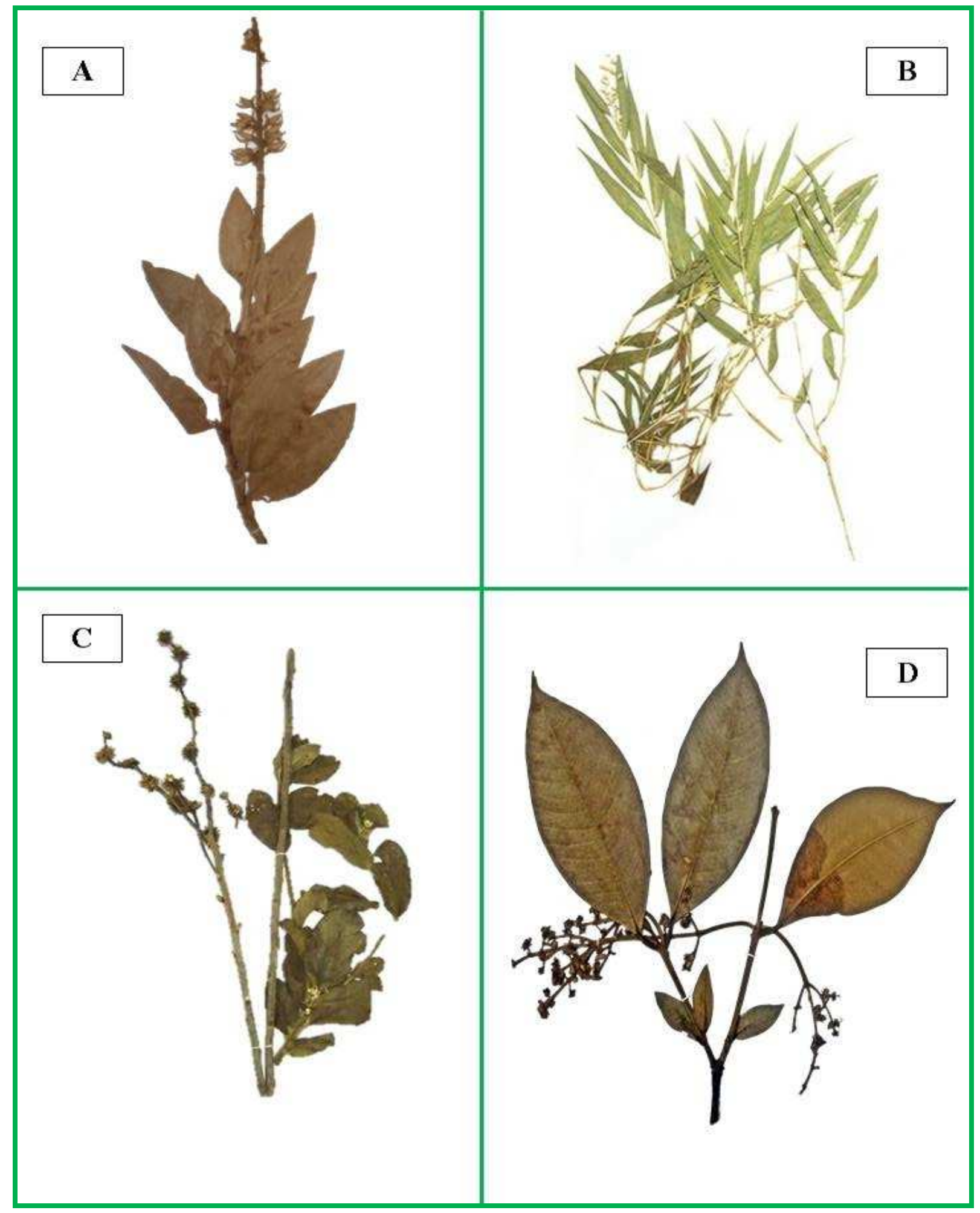

Figura 4: Representantes das famílias com maior número de espécimes doados ao herbário Professor Aluísio Bittencourt. A) Lupinus L. (Fabaceae) doado do Distrito Federal; B) Lasiacis (Griseb.) Hitchc. (Poaceae) doado do Distrito Federal; C Vernonia Schreb. (Asteraceae) doado de Minas Gerais e em D) Myrcia sellowiana O. Berg (Myrtaceae) doado do Distrito Federal. 